\title{
Diffuse optical tomography through solving a system of quadratic equations without re-estimating the derivatives: The "Frozen-Newton" method
}

\author{
B Kanmani* \& R.M.Vast \\ Department of Instrumentation \\ Indian Institute of Science, Bangalore India \\ E-mail: vasu@isu.iisc.emet.in \\ * On study leave from B.M.S.College of Engineering, Bangalore
}

\begin{abstract}
Optical tomography (OT) recovers the cross-sectiona distribution of optical paraneters inside a highly scattering medium from information contained in measurements that are performed on the boundary of the medium. The image reconstruction problem in OT can be considered as a large-scale optimization problem, in which an appropriately defined objective functional needs to be minimized. Most of earlier work is based on a forward model based iterative image reconstruction (MOBIIR) method. In this method, a Taylor series expansion of the forward propagation operator around the initial estimate, assumed to be close to the actual solution, is terminated at the first order term. The linearized perturbation equation is solved iteratively, re-estimating the first order term (or Jacobian) in each iteration, until a solution is reacbed. In this work we consider a nonlinear reconstruction problem, which has the second order term (Hessian) in addition to the first order. We show that in OT the Hessian is diagonally dominant and in this work an approximation involving the diagonal terms alone is used to formulate the nonlinear perturbation equation. This is solved using conjugate gradient search (CGS) without re-estimating either the Jacobian or the Hessian, resulting in reconstructions better than the origial MOBIIR reconstruction. The computation time in this case is reduced by a factor of three.
\end{abstract}

\section{INTRODUCTION}

There has been rapid development of noninvasive imaging modalities based on illumination with near-infrared light (NIR) for medical diagnosis, because of the relatively low absorption of such light by human tissue $[1,2]$. Breast cancer detection and monitoring of neonatal head for haemorrage are two important applications. The imaging is based on the recovery of two optical property distributions in the tissue, absorption $\left(\mu_{\mathrm{a}}\right)$ and scattering coefficient $\left(\mu_{\mathrm{s}}\right)$. by solving an inverse light propogation problem through the tissue. Inversion is usually done iteratively, by repetitive employment of a forward model of light propagation [3]. The radiative transfer equation (RTE) is an accurate model for photon transport using the particle picture of light. Numerical implementations of the RTE using finite element method (FEM) [4] or using finite difference discrete ordinate method (DOM) have been employed to solve both the forward [5] and inverse problem $[6,7]$ of optical tomography (OT). The Monte Carlo (MC) simulation [8], is another approach to transport photons through scattering media, which is a stochastic implementation of the RTE. Whereas the RTE and MC score on accuracy, they perform poorly when it comes to speed of implementation. Therefore an approximation to the RTE known as the diffusion approximation (DA) is made use of in both the forward and inverse problems of OT, with validity limited to cases where $\mu_{\mathrm{s}} \gg \mu_{\mathrm{a}}$ and away from sources and detectors. Even with this simplified model the inverse problem of OT is still computationally intensive. Optical property reconstruction has been performed by repeatedly solving the forward problem. All of the published work has used only a linear perturbation model [ 11] which used only the first order derivatives i.e., the Jacobian.

In this work we use a non-linear approximation of the perturbation equation by adding the second term involving the Hessian in the Taylor expansion. In the work reported in [12], it is shown that the overall inversion process required only one evaluation of both the Jacobian and the Hessian; in the context of the OT problem, this means, that the forward operator is expanded only once and approximated by the first and second order terms. The resulting set of non-linear equations is solved iteratively. This method is called the "Frozen-Newton" method [12] because the derivatives are evaluated only once in the beginning. We prove the efficacy of this method to solve the non-linear problem of OT.

\section{THE MOBIIR ALGORITHM}

The object is illuminated by a set of light sources from the boundary sequentially. Given the tissue parameter $\left\{\mu_{\mathrm{a}}, \mu_{\mathrm{s}}{ }^{\prime}\right\}$ distribution within the object, finding the resulting measurement $\mathbf{M}^{e}$ everywhere, especially on the boundary constitutes the forward problem, and can be expressed using a general non-linear forward operator $F$

$$
\mathbf{M}^{\boldsymbol{e}}=\boldsymbol{F}\left\{\mu_{\mathrm{a}}, \mu_{\mathrm{s}}\right\}
$$

On the other hand, given the source detector distribution, and the measurement set $\mathbf{M}^{e}$ on the

0-7803-8665-5/04/\$20.00 C2004 IEEE 
boundary, predicting the tissue parameter distribution $\left\{\mu_{\mathrm{a}}, \mu_{\mathrm{z}}{ }^{\prime}\right\}$ within the object, is the inverse problem where $\mu_{\mathrm{a}}$ is the absorption coefficient (in $\mathrm{cm}^{-1}$ ), $\mu_{\mathrm{s}}$ the scattering coefficient ( in $\mathrm{cm}^{-1}$ ) and $\mu_{s}^{\prime}$ is the reduced scattering coefficient (equal to $(1-\mathrm{g}) \mu_{\mathrm{s}}$ with $g$ being the anisotropy factor). This inverse problem, is highly non-linear, ill-posed, and its discretized version involves huge sparse matrices. Of the various methods available for solving the inverse problem, the model based iterative inverse reconstruction (MOBIR) [3] algorithm is widely used. This method involves repeated solution of the forward problem, and by comparison of the computed data set with the experimental data set, the minimization of a proper objective functional on the difference between the two. A brief description of the steps involved in the MOBMR procedure is given below

\section{The MOBIIR algorithm}

Given: The simulated experimental data set $-\mathbf{M}^{e}$

Stepl: Assume initial estimate $\mathbf{X}_{0}$ of parameter $\left\{\mu_{\mathrm{a}}, \mu_{2}\right\}$

Step 2: Obtain computed data set $\mathbf{M}^{\boldsymbol{c}}$ by applying the forward operator $F$ on $X_{a}$ =

Step 4: Obtain the difference $\Delta \mathbf{M}=\mathbf{M}^{e}-\mathbf{M}^{c}$

Step 5: Evaluate the Sensitivity matrix or the Jacobian J

Step 6: Determine incremental update $\Delta \mathbf{X}$ by solving the following perturbation equation (this is an iterative process)

$$
\mathbf{J}^{\mathbf{T}} \Delta \mathbf{M}=\mathbf{J}^{\mathbf{T}} \mathbf{J} \Delta \mathbf{X}
$$

Step 7: Update estimate by $\mathbf{X}=\mathbf{X}_{0}+\Delta \mathbf{X}$.

Repeat Step2 to 7 until solution converges indicated by $\left\|\mathbf{M}^{e}-\mathbf{M}^{e}\right\|<\delta$ where $\delta$ is predefined

This MOBIIR algorithm is based on the Taylor series expansion of operator $F$ given by

$\Delta \mathbf{M}=\Delta \mathbf{X} \boldsymbol{F}^{\prime}\left\{\mathbf{X}_{0}\right\}+\Delta \mathbf{X}^{\mathbf{T}} \boldsymbol{F}^{\prime \prime}\left\{\mathbf{X}_{\mathrm{p}}\right\} \Delta \mathbf{X}+$

where $\vec{F}^{\prime}$ and $\vec{F}^{\prime}$ are the Frechet derivatives, and are known as the Jacobian and the Hessian in the discrete domain case. The above equation can now be expressed in terms of the Jacobian $J$ and the Hessian H

$\Delta \mathbf{M}=\mathbf{J} \mathbf{\Delta X}+\Delta \mathbf{X}^{\mathbf{T}} \mathbf{H} \mathbf{\Delta X}+\ldots$

It can be clearly seen that the equation in step 6 of the MOBIIR algorithm, only the first term of Eq. (3) is used. We would like to highlight that, Eq. (1) is a linear approximation to the perturbation equation derived from the forward propagation operator. In iterative reconstruction, after each iruner iteration (represented by solution of Eq. (1) in step 6) the linearization has to be redone by recalculating Jacobian. In our modification described below, the linear approximation of Eq.(1) is replaced by Eq.(3) which includes the quadratic term as well.

\section{MODIFIED MOBIIR ALGORITHM.}

The new perturbation equation is

$$
\Delta \mathbf{M}=\mathbf{M}^{e}-\mathbf{M}^{c}=\mathbf{J} \Delta \mathbf{X}+\Delta \mathbf{X}^{\mathbf{T}} \mathbf{H} \boldsymbol{\Delta X}
$$

where $\mathbf{H}$ is the Hessian. The last term on the right hand side of Eq.(4) is the non-linear term involving the Hessian. In the modifted method this nonlinear perturbation equation is solved iteratively. We first discuss the evaluation of the Jacobain, followed by the evaluation of the Hessian and then the frozen-Newton algorithm involving repeated solution of Eq (4)with Jacobian and Hessian kept "frozen" at their initial estimated values.

\section{(a) Calculation of Jacobian}

The Jacobian is the first order derivative of the forward operator with respect to the optical parameters, in the discretized domain. We now evaluate the Jacobain for a single source-detector pair. The method can be easily extended to multiple source-detector pairs. Let the domain be discretized into E. non-overlapping elements connected by $\mathbf{N}$ nodes. Let $\phi_{\text {hom }}$ be the photon density at the detector with a delta source at the source position with the domain having homogeneous optical properties. Now introduce a small perturbation $\Delta p$ in the properties of element \#1. Compute the photon density $\phi_{\text {pert-1 }}$. Then the Jacobain for element \#lfrom basic principles ( as $\Delta p$ tends to zero) is identified as

$$
J_{1}=\left(\phi_{\text {tomo }}-\phi_{\text {pert-1 }}\right) / \Delta p
$$

We repeat this procedure for all elements in the domain to get the complete $(\mathbf{1 x N})$ vector of the Jacobian J. Evaluation of Jacobian by this method is form basic principles and is computationally intensive. A quick method of evaluating the Jacobian by evaluating the adjoint operator of the forward problem is given in references [9]-[11].

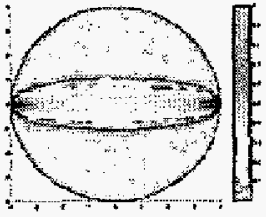

(a)

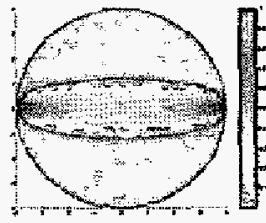

(b)
Figure 1: The Jacobian evaluated by (a) basic method, (b) adjoint operator

To obtain the Jacobian, we considered an object with a circular cross-section of diameter $8 \mathrm{~cm}$, with absorption coefficient of $0.25 \mathrm{~cm}^{-1}$ and scattering coefficient of $20 \mathrm{~cm}^{-1}$ with anisotropy factor of 0.9 and a source-detector separation of $180^{\circ}$. The domain is discretized into 2880 elements connected by 1501 nodes. All simulations are performed on a P-III computer with a $1.2 \mathrm{GHz}$ processor. Figure $1 \mathrm{a}$ gives the Jacobian evaluated by the basic method, and Figure $1 \mathrm{~b}$ gives the Jacobian evaluated by using the adjoint operator. We find that both methods yield 
similar results. However, the basic method took 680 seconds, while the adjoint method took 4 seconds.

\section{(b) Calculation of Hessian}

The Hessian is the second order derivative of the measurements with respect to the optical parameters, in the discretized domain. We now evaluate the Hessian for a single source-detector pair. The method can be easily extended to multiple source-detector pairs. Let $\mathbf{J}_{\text {homo }}$ be the Jacobian (vector of size $\mathbf{N}$ ) for this source-detector pair with domain having homogeneous optical properties. Now introduce a small perturbation $\Delta p$ in the properties of element \#1. Compute the Jacobian $\mathbf{J}_{\text {pert-1 }}$. Then the Hessian for element $\# 1$ from basic principles is identified as

$$
\mathbf{H}_{1}=\left(\mathbf{J}_{\text {homo }}-\mathbf{J}_{\text {pert-1 }}\right) / \Delta p
$$

The elements of $\mathbf{H}_{1}$, which are second derivatives (i.e., rate of change of the Jacobian elements with respect to change in $\mu_{2}$ or $\mu_{s}$ " of the element under consideration) is a vector of size $\mathbf{N}$. We repeat this procedure for all elements in the domain to get the complete (NxN) matrix of the Hessian $\mathbf{H}$.

Since, size of $\mathbf{H}$ is very large, to display as a singe full matrix is cumbersome, we study the statistics of the diagonals of the Hessian. Figures $2 a \& b$ give plots of the mean and variance of the band of diagonals up to 250 in one direction starting from the central diagonal. From this plot it is evident that $\mathbf{H}$ can be represented as a banded matrix and it is diagonally dominant. It was also found that the main diagonal of the Hessian has variation similar to the Jacobian. In our reconstruction algorithm, the Hessian is approximated as a diagonal matrix with values proportional to the element in the Jacobian. With this approximation, we iteratively solve the set of non-linear equations of Eq. (4).
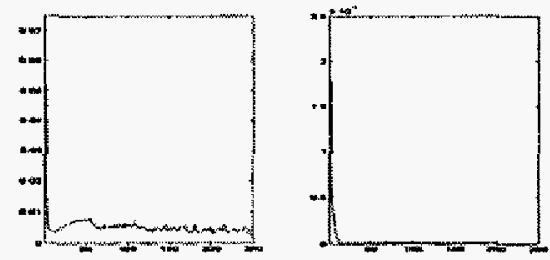

Figure 2: Plots of the mean and variance of the band of diagonals up to 250 in one direction starting from the central diagonal of the Hessian

\section{(c) The Frozen-Newton algorithm}

As mentioned earlier, the MOBIIR reconstruction algorithm is a linear iterative process, where the Jacobian is evaluated afresh at each iteration. Moreover, the algorithm works only for small perturbations in the background optical properties. Since it is a linear approximation to the highly non-linear OT reconstruction problem, it cannot reconstruct huge variations in optical parameters. The modified MOBIIR algorithm, because of the nonlinear term can handle larger variation in optical properties in the inhomogeneities. In addition, the derivatives, both Jacobian and Hessian, are evaluated only once in the beginning, (the "Frozen-Newton" method [12] ) and the perturbation equation is iteratively solved. The steps in the modified algorithm developed are as follows:-

\section{The non-linear algorithm}

Given: The simulated experimental data set $\mathbf{M}^{e}$

Step1: Assume initial estimate $\mathbf{X}_{\mathbf{0}}$ as the background optical property

Step 2: Evaluate the Jacobian $\mathbf{J}$ and Hessian $\mathbf{H}$ using $\mathbf{X}_{\mathbf{0}}$

Step 3: Obtain computed data set $\mathbf{M}^{e}$ by applying the forward operator $F$ on $\mathbf{X}_{\text {o }}$

Step 4: Obtain the difference $\Delta \mathbf{M}=\mathbf{M}^{e}-\mathbf{M}^{\boldsymbol{c}}$

Step 6: Solve for incremental update $\Delta \mathbf{X}$ by solving the following equation, using any standard non-linear optimization technique (this is an iterative process)

$$
\Delta \mathbf{M}=\Delta \mathbf{X} \mathbf{J}+\Delta \mathbf{X}^{\mathbf{T}} \mathbf{H} \Delta \mathbf{X}
$$

Step 7: Update estimate by $\mathbf{X}=\mathbf{X}_{\mathbf{0}}+\Delta \mathbf{X}$

Repeat Step 3 to 7 until solution converges indicated by $\left\|\mathbf{M}^{e}-\mathbf{M}^{e}\right\|<\delta$ where $\delta$ is predefined

\section{RECONSTRUCTION USING NUMERICAL PHANTOMS.}

To demonstrate our algorithm, we considered a 2-d object, with a circular cross-section of diameter $8 \mathrm{~cm}$, with background optical properties given by, absorption coefficient of $0.25 \mathrm{~cm}^{-1}$ and scattering coefficient of $20 \mathrm{~cm}^{-1}$ with anisotropy factor of 0.9 . Two inhomogeneities in absorption coefficient are introduced, one located at a distance of $2 \mathrm{~cm}$ from the center, with radius $0.8 \mathrm{~cm}$ with $\mu_{\mathrm{a}}^{\text {in }}=0.75 \mathrm{~cm}^{-1}$ and another at a distance of $2 \mathrm{~cm}$ (diametrically opposite the earlier inhomogeneity) from the center with radius $0.6 \mathrm{~cm}$ with $\mu_{\mathrm{n}}^{\text {in }}=0.5 \mathrm{~cm}^{-1}$. The domain is again discretized into 2880 elements connected by 1501 nodes. For data collection we used 12 source locations distributed around the object at angular spacing of $30^{\circ}$ each. For each source location there are 13 detectors equally spaced at $10^{\circ}$ apart arranged on the boundary of the object on the other side of the detector so that the total angle spanned by the detectors with respect to the source is $120^{\circ}$. Our initial guess in all cases is the background optical property. For the iterative process in step 6 of the MOBIIR algorithm and in the non-linear algorithm we used the conjugate gradient search (CGS) method. We give results of reconstruction using the two methods. 
(a) Reconstruction using the standard MOBIIR algorithm

The standard. MOBIIR algorithm is implemented. The program performed 21 iterations, taking a total time of 46 minutes. However the algorithm converged at the end of $13^{\text {th }}$ iteration itself. The reconstructed image showing absorption coefficient is shown in. Figure 3a. Figure 3b gives the cross-sectional plots through the inhomogeneities of Fig.3a. The results show that although the two inhomogeneities have been identified, the algorithm has not recovered the exact variations in the inhomogeneities.

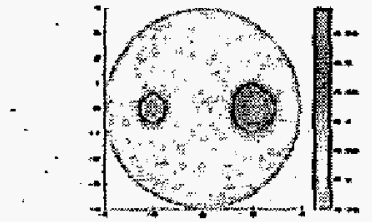

(a)

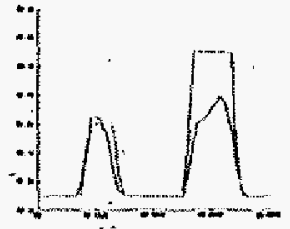

(b)
Figure 3: Reconstruction using the standard MOBLR method (a) the image, (b) the cross-sectional plots through the inhomogeneities

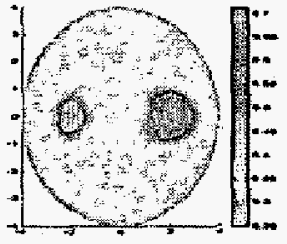

(a)

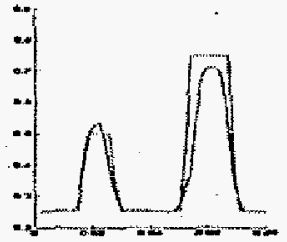

(b)
Figure 4: Reconstruction using the "Frozen-Newton" algorithm (a) the image, (b) the cross-sectional plots through the inhomogeneities

\section{(b) Reconstruction using "Frozen--Newton" algorithm}

We now implement the non-linear "frozen"-Newton algorithm as described in Section III. Here we compute the Jacobian and the Hessian for background optical properties. We approximate the Hessian as a diagonal matrix, proportional to the Jacobain. These derivatives are "frozen". In this algorithm, convergence, is obtained at the end of $16^{\text {th }}$ iteration. The total time taken is 16 minutes. The results of reconstruction is given in Figure $4 a, b$. The advantage of non-linear optimization including the Hessian is clearly evident. Of all the three methods, this algorithm gives best reconstruction results, as evidenced by the smallest error between $\left\|\mathbf{M}^{e}-\mathbf{M}^{c}\right\|$ consuming minimum time.

\section{CONCLUSIONS.}

We have evaluated the Hessian from basic principles, and, proved that the simplest approximation to the Hessian where it is represented as a diagonal matrix is good enough for use in a non-linear reconstruction algorithm. Since it is found that the diagonal terms of the Hessian are proportional to the terms of the Jacobian it is enough to put the concatenated Jaocobian in place of the Hessian diagonal in the iterative reconstruction procedure. Evaluation of the Jacobian is a well established process. The advantage of including the Hessian is that the Jacobian and the Hessian need not be recalculated at each iteration. From the numerical simulation example considered, we show that the modified MOBIIR results in quicker and better quality reconstruction of optical properties.

\section{References}

[1] Hebden J C, Arridge S R and Delpy D T, "OpticaI imaging in medicine: $I$. Experimental techniques" Phys. Med. Biol. 42, pp 825-840, 1997

[2] Arridge S R and Delpy D T, "Optical imaging in medicine: II. Modelling and reconstruction" Phys. Med. Biol. 42, pp 841-853, 1997

[3] Hielscher A H, Klose A D and Hansen $\mathbf{K ~ M}$, "Gradient-based iterative image reconstruction scheme for time-resolved optical tomography". IEEE Trons. Med. Imag. 18, pp 262-271, 1999

[4] Arridge $S R$, Schweiger $M$, Hiraoka $M$ and Delpy $D$ $\mathrm{T}$, "Finite element approach for modelling photon transport in tissue" Med. Phys. 20, pp 299-309, 1993

[5] - Klose A D, Netz U, Beutham J and Hielscher A H, "Optical tomography using the time-independent equation of radiative transfer: Part I. Forward model" J. Quant. Spectroc. Radiat. Transfer 72, pp 691-713, 2002

[6] Klose A D and Heilcher A H, "Optical tomography using time independent equation of radiative transfer: Part II. Inverse model". $J$. Quant. Spectroc. Radiat. Transfer 72, pp715-732, 2002

[7] Klose A D and Hielscher A H, "Iterative reconstruction scheme for optical tomography based on the equation of radiative transfer", Med. Phys. 26, pp 1698-1707, 1999

[8] Wang L and Jacques S L , "Monte Carlo modelling of light transport in multi-layered tissues, in standard C" (University of Texas M.D. Anderson Cancer Centre, Houston Texas) http://ecc.ogi.edu/omlc /science /software /mc/index.html 1992-93

[9] Arridge S R, "Photon-measurement density fuctions.Part I: Analytical forms" Appl. Opt. 34, pp 7395-409, 1995

[10] Arridge $S$, "Photon-measurement density functions. Part II: Finite-element-method calculations" Appl. Opt. 34, pp 8026-37, 1995

[11] Arridge $\mathbf{S} \mathbf{R}$, "Topical review: Optical tomography in medical imaging", Inverse Problems 15 R41-R93, 1999

[12] Hettlich F and Rundell W, "A second degree method for nonlinear inverse problems", Society for Industrial and Applied mathematics, Vol 37; No.2, pp 587-620, 2000 